\title{
Barriers to integrating personalized medicine into clinical practice: a best-worst scaling choice experiment
}

\author{
Mehdi Najafzadeh, MSc'1, Larry D. Lynd, PhD ${ }^{1,2}$, Jennifer C. Davis, PhD³, Stirling Bryan, $\mathrm{PhD}^{3,4}$, \\ Aslam Anis, PhD ${ }^{2,4}$, Marco Marra, PhD ${ }^{5}$ and Carlo A. Marra, PharmD, PhD ${ }^{1,2}$
}

\begin{abstract}
Purpose: As advances in genomics make genome sequencing more affordable, the availability of new genome-based diagnostic and therapeutic strategies (i.e., personalized medicine) will increase. This wave will hit front-line physicians who may be faced with a plethora of patients' expectations of integrating genomic data into clinical care. The objective of this study was to elicit the preferences of physicians regarding applying personalized medicine in their clinical practice as these strategies become available.
\end{abstract}

Methods: Using a best-worst scaling (BWS) choice experiment, we estimated the relative importance of attributes that influence physicians' decisions for using personalized medicine. Six attributes were included in the BWS: type of genetic tests, training for genetic testing, clinical guidelines, professional fee, privacy protection laws, and cost of genetic tests. A total of 197 physicians in British Columbia completed the experiment. Using latent class analysis (LCA), we explored the physicians' heterogeneities in preferences.
Results: "Type of genetic tests" had the largest importance, suggesting that the physicians' decision was highly influenced by the availability of genetic tests for patients' predisposition to diseases and/or drug response. "Training" and "guidelines" were the attributes with the next highest importance. LCA identified two classes of physicians. Relative to class 2, class 1 had a larger weight for the "type of genetic tests," but smaller weights for "professional fee" and "cost of tests."

Conclusion: We measured relative importance of factors that affect the decision of physicians to incorporate personalized medicine in their practice. These results can be used to design the policies for supporting physicians and facilitating the use of personalized medicine in the future.

Genet Med 2012:14(5):520-526

Key Words: best-worst scaling; discrete choice experiment; genomics; personalized medicine; preference elicitation

\section{INTRODUCTION}

With the availability of new technologies, the cost and time needed for complete sequencing of an individual's genome is rapidly declining. ${ }^{1,2}$ In 2007, Knome Incorporated announced the sequencing of an individual's entire genome for US $\$ 350,000$. Only three years later, in June 2010, Illumina announced it was providing the same service for $<\mathrm{US} \$ 9,500$, almost 35 times lower, using its new HiSeq2000 technology. ${ }^{3}$ In addition to the rapidity with which the entire genome can be sequenced, it is expected that it will cost $<\$ 1,000$, making it feasible for individuals to pay out-ofpocket to have their own personal genome sequenced. ${ }^{1,3-5}$

Despite these advances, there remains the challenge of decoding and interpretation of the data in the DNA sequences that are generated. The application of these data to the practice of clinical medicine and integration into patient care, often referred to as "personalized medicine," ${ }^{3}$ still requires a great amount of research in basic and applied sciences. ${ }^{7}$ For example, welldesigned clinical trials are still needed to establish the clinical validity of genetic tests due to the complexities of the interaction between the genes and the environment, and the interaction of multiple genes associated with different diseases.
Early signs of the therapeutic potential of personalized medicine are already visible in clinical practice and in developments led by the pharmaceutical industry. For example, panitumumab, a drug for the treatment of colon cancer, was shown to be effective only in cases without KRAS mutation in the tumor. Therefore, we could prevent inappropriate treatment of the other patients, who are not expected to experience a benefit. As another example, abacavir, an anti-HIV drug, results in serious side effects among patients with the HLA-B5701 allele, making prospective pharmacogenetic testing a realistic strategy for avoiding these adverse events..$^{8-10}$ These examples demonstrate how genetic testing facilitates tailored treatments, leading to greater effectiveness and/or fewer adverse effects. Further, genetic testing may be useful for predicting genetic risk for different diseases-i.e., as a surveillance tool.

As advances in genomics make genome sequencing more affordable, the demand for having these data, with the intent of using the information to inform clinical practice, will increase. This wave will hit front-line physicians who may be faced with a plethora of patients' expectations for the integration of genomic data into clinical care. Discoveries based on human genome

\footnotetext{
${ }^{1}$ Faculty of Pharmaceutical Sciences, University of British Columbia, Vancouver, British Columbia, Canada; ${ }^{2}$ Centre for Health Evaluation and Outcome Sciences, St. Paul's Hospital, Vancouver, British Columbia, Canada; ${ }^{3}$ Centre for Clinical Epidemiology and Evaluation, VCH Research Institute, The University of British Columbia, Vancouver, British Columbia, Canada; ${ }^{4}$ School of Population and Public Health, University of British Columbia, Vancouver, British Columbia, Canada; ${ }^{5}$ Genome Sciences Centre, Vancouver, British Columbia,

Canada. Correspondence: Carlo A. Marra (carlo.marra@ubc.ca)
} 
sequencing will increase the degree of complexity in diagnosis of diseases and their corresponding interventions. For example, different therapeutic approaches will be available depending on a patient's genotype, which will complicate the task of physicians and other health-care providers who deliver diagnostic or treatment services.

Once the developments in genomics are advanced enough for application in a clinical setting, we need to assess physician's readiness and willingness to use these new genetic tests. There are few studies that have investigated the role of primary-care physicians in the development and application of personalized medicine to routine clinical care. Most of these studies, with the exception of a few surveys, are qualitative in nature (interviews, focus groups, etc.) and have not been supplemented by quantitative data. ${ }^{11-21}$

The iGene study was intended to measure the relative importance of the barriers to integrating personalized medicine into practice from physicians' perspective. The novelty of this study is its quantitative approach using a best-worst scaling (BWS) choice experiment to answer this question and extend previous findings from the qualitative studies. As such, we combined the strengths of qualitative and quantitative methods for hypotheses generation and hypothesis testing, respectively.

\section{MATERIALS AND METHODS \\ Study design}

This study used a cross-sectional experimental design in which a sample of physicians in British Columbia provided their opinions using a computer-administered questionnaire. We used a BWS choice experiment for estimating relative importance of attributes that might affect a physician's decision to utilize personalized medicine in his/her practice.

The attributes included in this experiment were identified through a qualitative study using several focus groups that included 28 physicians in British Columbia, Canada. We conducted three semi-structured focus groups in two urban (Fraser, Vancouver Coastal Health) and one rural (Interior) setting in British Columbia with 28 physicians to facilitate the selection of attributes for the choice experiment. We used the constant comparative method and we did a line-by-line analysis to identify words, phrases, and concepts with higher importance. We identified several themes that were discussed in subsequent focus groups. Finally, we hand-coded the data and selected the dominant themes and important attributes that emerged from the focus group. Table 1 shows the list of attributes that emerged as important items during the discussions in the focus

\section{Table 1 Attributes and levels included in the best-worst questionnaire}

\section{Type of genetic tests}

Both types: Most of the available genetic tests would be intended to specify both patients' predispositions to diseases and patients' drug responses based on their genotypes.

Tests for disease predisposition: Most of the available genetic tests would be intended to specify patients' predispositions to various diseases. Tests for drug response: Most of the available genetic tests would be intended to specify more effective drugs and/or drugs with less adverse effects for specific diseases based on patients' genotypes.

\section{Training for the use of genetic testing}

Extensive training opportunities: 3 hour hands-on workshop for each new genetic test

Some training opportunities: 1 hour in-service for each new genetic test.

No training opportunity: No particular training opportunity would be provided for physicians about the use of new genetic tests.

\section{Guidelines for the use of genetic testing}

Clear guidelines: There would be clear and comprehensive clinical guidelines for the use of genetic tests

No guidelines: There would not be any clinical guidelines for the use of genetic tests.

\section{Professional fee}

\$100: A \$100 professional fee would be defined in payment schedules (similar to the fee for performing a cardiovascular risk assessment) for using a genetic test.

\$16: A \$16 professional fee would be defined in payment schedules (similar to electrocardiogram tracing and interpretation fee) for using a genetic test.

\$0: No professional fee would be added to payment schedules.

\section{Privacy protection laws}

Comprehensive protection laws: There would be comprehensive and effective laws and regulations to protect the privacy of the patients' genetic information.

No protection laws: There would be no particular laws or regulations to protect the privacy of the patients' genetic information.

\section{Cost of genetic tests}

\$0: Medical insurance plans would cover $100 \%$ of the expense of the genetic tests that are shown to be cost effective.

$\$ 250$ : A typical genetic test would cost about $\$ 250$ and it usually would not be covered by health insurance.

$\$ 500$ : A typical genetic test would cost about $\$ 500$ and it usually would not be covered by health insurance. 
groups. Each attribute can assume a possible level (referred to as attribute levels from here on).

Choice experiments are particularly useful in measuring preferences about the options that are on the horizon of clinical innovation. ${ }^{22-25}$ Genetic tests that have not been implemented yet are a good example of this application. ${ }^{26,27}$ BWS is a cuttingedge method for conducting choice experiments and has several advantages over older methods. ${ }^{28,29}$ One of the key advantages of BWS is the ability to estimate the relative importance of all attributes on a common scale, which is crucial for the purposes of our study.

\section{Questionnaire design}

BWS is a method of eliciting the individuals' stated preferences for a specific set of attributes or characteristics associated with a decision or choice. ${ }^{29}$ In BWS experiments, each respondent chooses the most preferred and the least preferred items among a list of three or more items presented in a given task. This process is then repeated, with each subsequent choice task containing a different set of items. This method ensures obtaining a valid estimation of the respondents' underlying relative preferences for the items presented. ${ }^{28,30}$ Considering this general framework, we designed a BWS experiment to measure preferences of physicians for the attributes of personalized medicine that may affect their decision to use it in their clinical practice. The questionnaire contained 16 tasks that had to be completed by each responder. Table 2 shows a sample task of the questionnaire.

Before completing the choice questionnaire, respondents were provided with two pieces of background information: (i) a brief introduction regarding the concept of personalized medicine; and (ii) a description of how personalized medicine is expected to enhance a physician's ability to prevent the occurrence of disease, and to diagnose and treat disease once it occurs. Consequently, participants completed the tasks under the assumption that genetic tests for which there was evidence of clinical benefits were available to be used in their clinical practice. Responders chose the attribute levels with the most positive effect and the most negative effect on their decision to use personalized medicine given the attribute levels presented in a given choice task. Physicians in Canada are generally being compensated according to provincial payment schedules for specific services or procedures. As such, the attribute

\section{Table 2 A sample best-worst choice task}

\begin{tabular}{|l|l|} 
Best & Worst \\
\hline & No training opportunity \\
\hline & Clear guidelines \\
& A $\$ 100$ professional fee \\
& Comprehensive protection laws \\
& Cost $\$ 250$
\end{tabular}

Each questionnaire consisted of 16 choice tasks. "professional fee" reflects the influence of monetary incentive on their decision to use genetic testing.

A balanced incomplete block design was used for the design of the consecutive tasks. ${ }^{31,32}$ Balanced incomplete block design ensures that all attributes and all pairs of attribute levels are presented with a balanced frequency to the responders. This property affirms an unbiased estimation of attribute scales. Each questionnaire was administered using a Web-based survey, and data were electronically collected on a secure server. Sawtooth software (Sawtooth Software, Sequim, WA) was used to generate the questionnaire and web page design, and to facilitate the collection and recording of responses into a secure database.

\section{Study sample}

The choice experiment involved the administration of a questionnaire to a sample of physicians registered with the BC College of Physicians and Surgeons. Physicians were contacted via e-mail and provided with a unique URL with password-protected identification embedded. A $\$ 50$ incentive was offered to participants upon completion of the questionnaire to compensate for the opportunity costs of participation in this survey, which took $\sim 30$ min to complete. A public market research firm screened physicians who met the inclusion criteria and was responsible for recruitment. The physicians in their database provided consent to be contacted for research studies. The sample of physicians who were contacted is a representative sample of family physicians and clinical geneticists in British Columbia.

Physicians' participation was optional, and they could choose to not answer any of the questions and/or withdraw at any time. The protocol for this study was reviewed and approved by the University of British Columbia Human Ethics Board, division of human behavioral studies.

\section{Statistical analysis}

BWS data were coded for latent class analysis (LCA) using Latent Gold Choice, version 4.5.0. Use of a conditional logit model can result in biased preference estimations if unknown correlation structures are present in the choice data. More sophisticated estimation methods such as LCA or, alternatively, mixed logit, are appropriate for modeling of choice data. ${ }^{33}$ LCA can also identify subgroups of individuals with a larger likelihood of fitting to a model, and in our case was an effective method to reveal heterogeneity of responders and their underlying preferences. ${ }^{33}$ We also included the characteristics of physicians in the LCA and tested for possible interaction of those characteristics with preference estimates.

The conventional methods for carrying out choice experiments, such as choice-based conjoint studies, facilitate the estimation of how moving between levels of an attribute affects overall utility. As the estimated model is essentially a difference model, estimated utilities measure only the utility of deviation from a reference level within each attribute. Therefore, it is not possible to compare the utility of levels between attributes. By contrast, BWS has a technical advantage that allows for the 
comparison of utilities across all levels of all attributes. Unlike choice-based conjoint, the utility of all attribute levels can be estimated relative to one reference attribute level, which is the only missing utility. As all utility estimates are then relative values in a common scale, it should be noted that shifting the horizontal axis in Figure 1, either upward or downward, will not affect the interpretation of the results. This study exemplifies a research question (i.e., relative importance of attributes) that cannot be properly answered using traditional methods of discrete choice experiments.

\section{Sample characteristics}

\section{RESULTS}

A total of 244 physicians were initially contacted, and 197 physicians completed the experiment and fully responded to the questionnaire (response rate $81 \%$ ). The mean age of the participants was 50.4 years (range 32-77 years), and on average they had 22 years of experience in clinical practice (Table 3 ). Approximately one-third of physicians who participated in this experiment were female (32\%) and the majority of them were practicing in urban areas in British Columbia (85\%).

\section{Model estimation}

The BWS method allows the estimation of coefficients for 15 attribute levels relative to the one specific level of one attribute. Thus, we estimated the preference for each level of attributes relative to the out-of-pocket "cost of a genetic test $=\$ 500$." As such, each coefficient can be considered to be the "utility" of that attribute-level on a common underlying preference scale. ${ }^{31}$ In addition, we calculated the impact of each attribute on the choices by averaging the coefficients of attribute levels for that attribute. The calculated attribute impacts can be interpreted as the relative importance of attributes. ${ }^{28,30,31}$

The estimated utilities of each attribute level and the relative importance of each attribute are reported in Table 4. The availability of "Both types" of genetic tests (genetic tests intended to

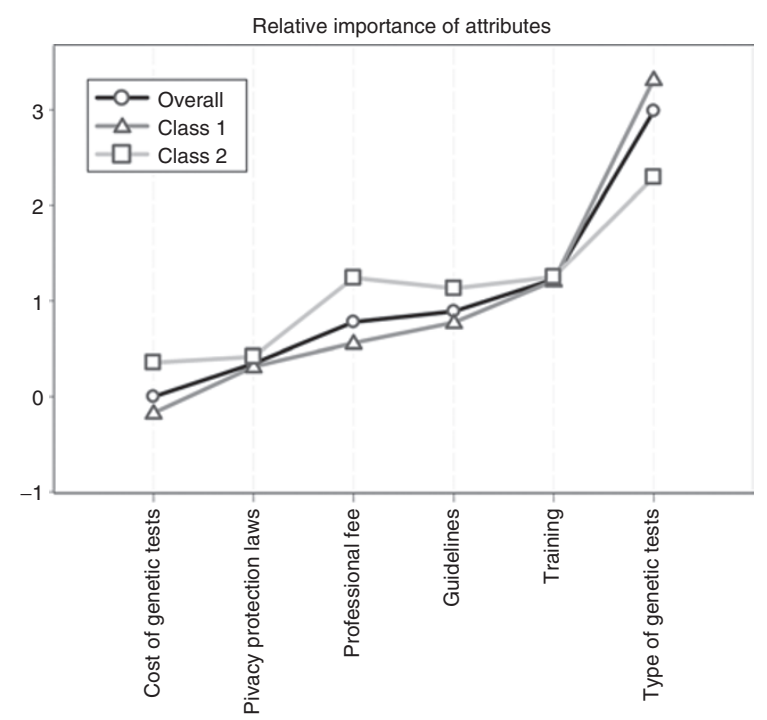

Figure 1 Relative importance of attributes. specify both patients' predispositions to diseases and patients' drug responses) and "Clear guidelines" were the most valued attribute levels, with mean utility estimates of 3.96 ( $P$ value $<0.001)$ and 3.79 ( $P$ value $<0.001)$, respectively. In comparison, utility estimates of "Extensive training opportunities" and a "\$100" professional fee were slightly lower, -3.03 ( $P$ value $<0.001)$ and $2.74(P$ value $<0.001)$ respectively. Conversely, "No guidelines," "No privacy protection laws," and a cost of “ $\$ 500$ " for a typical genetic test had the lowest utility estimates, -2.01 ( $P$ value $<0.001),-1.91$ ( $P$ value $<0.001)$, and -1.74 ( $P$ value $=1$ ), respectively. Utility estimates for "No training opportunity" and "No professional fee" were $-1.52(P$ value $<0.001)$ and $-0.91(P$ value $<0.001)$, respectively, suggesting their less detrimental effect on physicians' decision to apply genetic testing.

\section{LCA}

The second and third columns in Table 4 compare the utility estimates of two latent classes. The availability of "Both types" of genetic tests had the largest positive impact on the utility of respondents in class 1 , whereas a " $\$ 100$ " professional fee had

Table 3 Characteristics of participants $(N=197)$

\begin{tabular}{|c|c|}
\hline \multicolumn{2}{|l|}{ Age (years) } \\
\hline Mean (s.d.) & $50.4(9.2)$ \\
\hline Range (min-max) & $32-77$ \\
\hline \multicolumn{2}{|l|}{ Practice experience (years) } \\
\hline Mean (s.d.) & $22.0(9.8)$ \\
\hline Range (min-max) & $0-40$ \\
\hline \multicolumn{2}{|l|}{ Gender $(N)$} \\
\hline Female & $63(32 \%)$ \\
\hline Male & $134(68 \%)$ \\
\hline \multicolumn{2}{|l|}{ Practice field $(N)$} \\
\hline Family physician & $182(92.4 \%)^{a}$ \\
\hline Specialist & $11(5.6 \%)$ \\
\hline \multicolumn{2}{|l|}{ Practice location $(N)$} \\
\hline Rural & $29(14.7 \%)$ \\
\hline Urban & $165(83.8 \%)$ \\
\hline \multicolumn{2}{|l|}{ Practice type $(N)$} \\
\hline Family physician group practice & $113(57.4 \%)$ \\
\hline Multidisciplinary group practice & $11(5.6 \%)$ \\
\hline Solo practice & $48(24.4 \%)$ \\
\hline Specialized clinic & $10(5.1 \%)$ \\
\hline Other & $13(6.6 \%)$ \\
\hline \multicolumn{2}{|l|}{ Practice setting $(N)$} \\
\hline Private office/clinic & $139(70.6 \%)$ \\
\hline Hospital & $24(12.2 \%)$ \\
\hline Community clinic/health center & $16(8.1 \%)$ \\
\hline Academic center & $7(3.6 \%)$ \\
\hline Nursing home & $6(3.0 \%)$ \\
\hline Other & $3(1.5 \%)$ \\
\hline
\end{tabular}


Table 4 Estimation results from latent class analysis-conditional logit model

\begin{tabular}{|c|c|c|c|c|c|c|c|}
\hline \multirow[b]{2}{*}{ Class size } & \multicolumn{2}{|c|}{ Overall, $197(100 \%)$} & \multicolumn{2}{|c|}{ Class 1, $135(68 \%)$} & \multicolumn{2}{|c|}{ Class 2, $62(32 \%)$} & \multirow[b]{2}{*}{$P$ value } \\
\hline & Coefficient $^{\mathrm{a}}$ & T-ratio & Coefficient & T-ratio & Coefficient & T-ratio & \\
\hline Type of genetic tests & 2.99 & - & 3.32 & - & 2.29 & - & - \\
\hline Tests for disease predisposition & 2.69 & 14.07 & 2.94 & 12.59 & 2.17 & 6.84 & 0.001 \\
\hline Tests for drug response & 2.31 & 10.97 & 2.63 & 11.03 & 1.65 & 4.18 & 0.001 \\
\hline Some training opportunities & 2.16 & 8.75 & 2.27 & 7.90 & 1.95 & 5.27 & 0.51 \\
\hline No training opportunity & -1.52 & -28.51 & -1.87 & -24.93 & -0.79 & -10.94 & 0.017 \\
\hline Guidelines for the use of genetic testing & 0.89 & - & 0.77 & - & 1.13 & - & - \\
\hline Clear guidelines & 3.79 & 24.28 & 4.16 & 20.07 & 3.03 & 10.26 & 0.35 \\
\hline$\$ 16$ & 0.50 & -5.05 & 0.46 & -2.52 & 0.60 & -1.37 & 0.001 \\
\hline$\$ 0$ & -0.91 & -21.09 & -0.85 & -12.93 & -1.04 & -11.33 & 0.001 \\
\hline Genetic information privacy protection laws & 0.35 & - & 0.31 & - & 0.42 & - & - \\
\hline Comprehensive protection laws & 2.60 & 13.37 & 3.23 & 14.53 & 1.30 & 2.07 & 0.001 \\
\hline No protection laws & -1.91 & -34.88 & -2.60 & -32.27 & -0.46 & -8.61 & 0.001 \\
\hline Cost of genetic tests & 0.00 & - & -0.17 & - & 0.36 & - & - \\
\hline$\$ 0$ & 1.95 & 7.52 & 1.91 & 6.04 & 2.02 & 5.75 & 0.65 \\
\hline$\$ 250$ & -0.21 & -11.78 & -0.43 & -10.57 & 0.26 & -3.91 & 0.001 \\
\hline$\$ 500$ & -1.74 & 0.00 & -1.99 & 0.00 & -1.21 & 0.00 & 1 \\
\hline Log-likelihood & $-6,430.4$ & - & - & - & - & - & - \\
\hline Bayesian information criterion & $10,165.03$ & - & - & - & - & - & - \\
\hline Prediction error & 0.40 & - & - & - & - & - & - \\
\hline
\end{tabular}

${ }^{a}$ Estimated utilities (relative preference weights). ${ }^{b} P$ value is based on Wald statistics under the null hypothesis that coefficients in two classes are equal.

the largest positive impact in class 2. Furthermore, the lowest utility estimates in class 1 were "No guidelines" and "No privacy protection laws," in contrast to " $\$ 500$ " cost of genetic testing and "No professional fee" in class 2 . In class 1 , the positive impact of "Comprehensive privacy protection laws" was clearly larger as compared with class 2 . The preference estimates in class 1 evidently cover a wider range, suggesting stronger preferences in class 1 as compared with class 2. Supplementary Figure $\mathbf{1} 1$ online provides a visual illustration of the estimated coefficients in Table 4.

Considering the relative importance of the attributes shown in Figure 1, "Type of genetic tests" was the most important attribute affecting physicians' decision to use genetic testing in their practice in both classes, with a higher magnitude in class 1 as compared with class 2 (3.32 and 2.29, respectively). The importance of other attributes was similar in both classes with the exception of "Professional fee" and "Cost of genetic tests," which had slightly greater importance in class 2.

As individuals' characteristics (i.e., age, experience, practice type, location, setting, and field) were not statistically significantly different between the two latent classes, class membership could not be explained based on their known characteristics. However, most female physicians belonged to class 1 , and the proportion of females in the two classes was statistically significantly different. 


\section{DISCUSSION}

This study measured the relative importance of factors that affect the decision of physicians to incorporate new approaches of personalized medicine into their practice as they become available. The type of genetic tests was the most important attribute (2.99), suggesting that overall, availability of genetic tests has the largest influence on physicians' decision to use personalized medicine. In comparison, privacy protection regulations and out-of-pocket cost of genetic tests were the attributes with the least importance. Providing a professional fee for using genetic tests had only a moderate impact on physicians' decisions.

Participants also indicated a very large importance for the presence of clear clinical guidelines for the use of genetic testing (3.79). At the same time, lack of clinical guidelines had the lowest utility estimate $(-2.01)$, suggesting that lack of guidelines was probably considered to be the largest barrier to using genetic testing. In the feedback that physicians provided at the end of their choice experiment, they occasionally commented that providing online resources can be a practical alternative to formal training sessions about genetic testing. Our results suggest that the lack of training opportunities had a smaller negative effect as compared with lack of guidelines, which essentially reflects their preference in this regard.

In general, physicians in class 1 tended to be more willing to become pioneers in using new genetic tests-if they were accessible-and they perceived the cost of genetic tests, privacy protection laws, and professional fee as the less important obstacles to using personalized medicine. In contrast, physicians in class 2 , which consists of a smaller group of mostly male physicians (32\%), put less emphasis on the availability of genetic tests and more emphasis on the professional fee and cost of genetic tests.

A few studies have explored physicians' viewpoints about integration of genomic medicine into clinical care using cross-sectional surveys. Wideroff et al..$^{19}$ used the results of a national survey in United States to determine prevalence of using cancer susceptibility tests by physicians and to assess demographic variables associated with their use. They suggested that validity of the test, confidentiality, and affordability are among concerns about genetic testing. Freedman and colleagues $^{17}$ have surveyed 1,251 physicians in the United States and studied the factors that affect physicians' opinions on using cancer susceptibility genetic tests. Among other factors, they emphasized having clinical guidelines and training of physicians about genetic testing. Suther et al. ${ }^{20}$ have shown how physicians' perceptions about the characteristics of genetic tests influence the likelihood of adopting genomic medicine in their practice. Finally, Levy et al. ${ }^{21}$ have conducted a mail survey of 562 physicians and measured the importance of eight factors influencing physicians' decisions to use a genetic test to tailor smoking cessation treatment. They concluded that although physicians are eager to use genetic testing, lack of antidiscrimination measures may have negative influences on their decisions to use genetic testing. Our study has used the results of those articles to measure relative importance of those factors.

For the first time, our study uses a quantitative approach that seeks to elicit the preferences of physicians about personalized medicine. We used a BWS choice experiment, which is the state-of-the-art method for measuring the relative importance of attributes. In a choice experiment, responders are asked to indicate their trade-offs between different attributes and as such, estimated preferences reflect their underlying stated preferences. ${ }^{29}$ This study exemplified how quantitative methods such as choice experiments are particularly useful for policy making and priority setting through measuring aggregated preferences. Although the major factors that influence physicians' decisions to use genetic testing in their practices have been explored in several previous studies using qualitative methods, the relative importance of those factors was unknown. For example, the larger importance of clinical guidelines relative to incentives based on the results from our study suggests that policy makers should plan to incorporate genomic testing components into the current clinical guidelines to support integration of genomics into clinical care. Therefore, knowledge about the relative importance of those factors can be used to prioritize the work that needs to be done to accelerate this process.

Choosing the appropriate levels of attributes to ensure full coverage of all possible attributes that might affect a physician's decision to use genetic test was a challenge in the questionnaire designing stage. Although we tried to benefit from the discussion in focus groups and previous literature to address this issue, achieving a simple questionnaire and reducing the number of attribute levels to the possible minimum forced us to make difficult trade-offs. This limitation is not unique to our study; most choice experiments are confined in terms of maximum number of attributes and levels that are included in the design, mostly to avoid detrimental complexity of choice tasks. Nonetheless, we have utilized focus groups to identify attributes with the largest influence on physicians' decisions, and ,we therefore expect that inclusion of other attributes would not have significant effects on the estimated utility weights.

Our entire sample is taken from British Columbia; therefore, generalizability of these finding into other jurisdictions is questionable. In addition, using a Web-based sampling approach might introduce some biases, especially if the physicians who did not participate in the experiment were essentially a different class of physicians in terms of their underlying preferences. However, parallel findings of the focus groups and a relatively large sample size with a wide range of characteristics suggest that this bias should be negligible.

The results of this study can inform decision makers who design guidelines for physicians and facilitate the use of personalized medicine in the coming years. Further, understanding of physicians' preferences about personalized medicine will help prepare our health-care system to respond in a way that maximizes the potential benefits of relevant applications of genomics. 


\section{SUPPLEMENTARY MATERIAL}

Supplementary material is linked to the online version of the paper at http://www.nature.com/gim

\section{ACKNOWLEDGMENTS}

This project has been supported by British Columbia Clinical Genomics Network. M.N. had full access to all the data in the study and takes responsibility for the integrity of the data and the accuracy of the data analysis.

\section{DISCLOSURES}

The authors declare no conflict of interest.

\section{REFERENCES}

1. Service RF. Gene sequencing. The race for the $\$ 1000$ genome. Science 2006;311:1544-1546.

2. Snyder M, Du J, Gerstein M. Personal genome sequencing: current approaches and challenges. Genes Dev 2010;24:423-431.

3. Genomeweb. As Illumina Cuts Price of Personal Sequencing Service, CEO Says Market Growth Hinges on Analysis. http://www.genomeweb.com/sequencing/ illumina-cuts-price-personal-sequencing-service-ceo-says-market-growthhinges-an. Accessed 18 August 2010.

4. ten Bosch JR, Grody WW. Keeping up with the next generation: massively parallel sequencing in clinical diagnostics. J Mol Diagn 2008;10:484-492.

5. Podolak E. Sequencing's new race. BioTechniques 2010;48:105-111.

6. Burke W, Psaty BM. Personalized medicine in the era of genomics. JAMA 2007;298:1682-1684.

7. Feero WG, Guttmacher AE, Collins FS. The genome gets personal-almost. JAMA 2008;299:1351-1352.

8. Allison M. Is personalized medicine finally arriving? Nat Biotechnol 2008;26:509-517.

9. Shin J, Kayser SR, Langaee TY. Pharmacogenetics: from discovery to patient care. Am J Health Syst Pharm 2009;66:625-637.

10. Ikediobi ON, Shin J, Nussbaum RL, et al.; UCSF Center for Translational and Policy Research on Personalized Medicine. Addressing the challenges of the clinical application of pharmacogenetic testing. Clin Pharmacol Ther 2009;86:28-31.

11. Scheuner MT, Sieverding P, Shekelle PG. Delivery of genomic medicine for common chronic adult diseases: a systematic review. JAMA 2008;299: 1320-1334.

12. Hindorff $L A, B$ Burke $W$, Laberge $A M$, et al. Motivating factors for physician ordering of factor $V$ Leiden genetic tests. Arch Intern Med 2009;169:68-74.

13. Carroll JC, Brown JB, Blaine S, Glendon G, Pugh P, Medved W. Genetic susceptibility to cancer. Family physicians' experience. Can Fam Physician 2003;49:45-52.

14. Ohata T, Tsuchiya A, Watanabe M, Sumida T, Takada F. Physicians' opinion for 'new' genetic testing in Japan. J Hum Genet 2009;54:203-208.
15. Kumar S, Gantley M. Tensions between policy makers and general practitioners in implementing new genetics: grounded theory interview study. BMJ 1999:319:1410-1413.

16. Wilkins-Haug L, Erickson K, Hill L, Power M, Holzman GB, Schulkin J. Obstetrician-gynecologists' opinions and attitudes on the role of genetics in women's health. J Womens Health Gend Based Med 2000;9:873-879.

17. Freedman $A N$, Wideroff $L$, Olson $L$, et al. US physicians' attitudes toward genetic testing for cancer susceptibility. Am J Med Genet A 2003;120A:63-71.

18. Gramling R, Nash J, Siren K, Culpepper L. Predictive genetics in primary care: expectations for the motivational impact of genetic testing affects the importance family physicians place on screening for familial cancer risk. Genet Med 2003;5:172-175.

19. Wideroff $L$, Freedman $A N$, Olson $L$, et al. Physician use of genetic testing for cancer susceptibility: results of a national survey. Cancer Epidemiol Biomarkers Prev 2003;12:295-303.

20. Suther S, Goodson P. Barriers to the provision of genetic services by primary care physicians: a systematic review of the literature. Genet Med 2003; 5:70-76.

21. Levy DE, Youatt EJ, Shields AE. Primary care physicians' concerns about offering a genetic test to tailor smoking cessation treatment. Genet Med 2007;9: 842-849.

22. McTaggart-Cowan HM, Shi P, Fitzgerald JM, et al. An evaluation of patients' willingness to trade symptom-free days for asthma-related treatment risks: a discrete choice experiment. J Asthma 2008;45:630-638.

23. Mentzakis E, Ryan M, McNamee P. Using discrete choice experiments to value informal care tasks: exploring preference heterogeneity. Health Econ 2011;20:930-944.

24. Ryan M, Farrar S. Using conjoint analysis to elicit preferences for health care. BMJ 2000;320:1530-1533.

25. Ryan M, Scott DA, Reeves C, et al. Eliciting public preferences for healthcare: a systematic review of techniques. Health Technol Assess 2001;5:1-186.

26. Grosse SD, Wordsworth S, Payne K. Economic methods for valuing the outcomes of genetic testing: beyond cost-effectiveness analysis. Genet Med 2008; 10:648-654.

27. Regier DA, Ryan M, Phimister E, Marra CA. Bayesian and classical estimation of mixed logit: An application to genetic testing. J Health Econ 2009;28: 598-610.

28. Flynn TN, Louviere JJ, Peters TJ, Coast J. Best-worst scaling: What it can do for health care research and how to do it. J Health Econ 2007;26:171-189.

29. Lancsar E, Louviere J. Conducting discrete choice experiments to inform healthcare decision making: a user's guide. Pharmacoeconomics 2008;26: 661-677.

30. Marley AAJ, Louviere JJ. Some probabilistic models of best, worst, and bestworst choices. J Math Psychol 2005;49:464-480.

31. Marley AAJ, Flynn TN, Louviere JJ. Probabilistic models of set-dependent and attribute-level best-worst choice. J Math Psychol 2008;52:281-296.

32. Louviere JJ, Street D, Burgess L, Wasi N, Islam T, Marley AAJ. Modeling the choices of individual decision-makers by combining efficient choice experiment designs with extra preference information. J Choice Model 2008;1:128.

33. Greene $W H$, Hensher DA. A latent class model for discrete choice analysis: contrasts with mixed logit. Transport Res B 2003;37:681-698. 